\title{
Pensar en el aula desde la investigación
}

\author{
Thinking in the classroom from research
}

\author{
Dra. Lya Sañudo Guerra*
}

Fecha de recepción: 29 de mayo de 2009

Fecha de revisión: 12 de junio de 2009

Fecha de aprobación: 12 de agosto de 2009

\section{Resumen}

En estos últimos años, la investigación como proceso de formación inicial y continua de docentes ha tenido una presencia progresiva en el ámbito académico. Este artículo recupera las tendencias internacionales más relevantes en este sentido. Se hace un especial énfasis en la investigación que tiene como fundamento la práctica reflexiva. Finalmente, introduce una reflexión sobre la posibilidad de la producción del conocimiento educativo como un área específica del saber.

Palabras clave: investigación, práctica reflexiva, educación, formación docente, producción de conocimiento.

Es investigadora en dos campos específicos: la práctica y el significado en educación y la investigación de la investigación. Afines a estos temas ha presentado conferencias, ponencias y realizado publicaciones en México y en diferentes países. Ha participado en el diseño curricular de diferentes programas de posgrado y ha fundado y colaborado en redes de vinculación interinstitucional, nacionales e internacionales. Es reconocida por el Sistema Nacional de Investigadores del CONACyT en México. Correos electrónicos: Iya.sanudo@jalisco.gob.mx, lyasa54@yahoo.com.mx 


\begin{abstract}
This article shows the narrow relationship between the purposes and objectives of the educational research, the necessity of an ethical reflection of the educational research as important in the same morals of the daily practices and the administration that it involves in a deeper way the political decisions; and the necessity to generate valid and significant changes. From above-mentioned it is integrated to the research administration and the promulgation of an ethical code of the educational research as social research, with emphasis in a qualitative posture of investigation that is considered needier of articulating decisions to a combination of ethics of the conviction with an ethics of the principle.
\end{abstract}

Key words: educational research, ethics of the research, utilitarianism, rights of third generation.

La experiencia estimula la indagación, y la indagación no es más que la inteligencia que se pone a sí misma en acto.

Lonergan, 1989

\section{Introducción}

La preocupación por la formación de docentes es común en centros educativos de cualquier nivel; las propuestas en este sentido son abundantes. La argumentada en este texto es definitivamente controversial y muy actual en las discusiones de educadores e investigadores. Se pretende hacer una revisión de las principales tendencias en la formación y desarrollo de profesionales de la educación y llegar finalmente a la conclusión de que las nuevas exigencias educativas comprometen al(a) educador(a) a mejorar permanentemente su hacer en el aula. Para eso, se articula la investigación a su ejercicio cotidiano como una de las alternativas más productivas.

Identificar históricamente las grandes tendencias sobre la formación ${ }^{1}$ de los profesionales de la educación es relativamente fácil dadas las características propias de cada una. La mayor parte de los modelos utilizados puede ser incluida en las tendencias identificadas por Sacristán (1997), como la técnica, la académica, la investigación de la práctica para la investigación social y la práctica reflexiva.

Las dos primeras, aunque todavía presentes en la mayoría de los modelos de formación, no son de interés para este trabajo. La perspectiva

1 Para este trabajo llamaremos formación a todas las acciones del profesional de la educación dirigidas a la formación o desarrollo profesional o perfeccionamiento. De acuerdo con Barbier (1993), los sujetos se encuentran en continua formación. Las acciones se diferencian de acuerdo con su intención, su sistematicidad y su intensidad. 
técnica es aquella en la que el o la docente debe prepararse en el dominio de técnicas y procedimientos para aumentar el rendimiento académico de los estudiantes. Es el caso de los cursos de didáctica, microenseñanza, dinámica de grupos o similares para docentes en funciones, en los cuales se les capacita sobre asuntos de corte técnico con el supuesto de que, a través de ellos, se mejora el trabajo del aula. En la mayoría de las propuestas desde esta orientación se describe con detalle lo que tradicionalmente se llama enseñanza y se pierde o es inexistente lo relacionado con el aprendizaje. Proponen mejorar cómo se enseña; lo que no está claro, es si con ello se mejora el aprendizaje.

Esta tendencia originalmente se ve impulsada por el nacimiento de la escolarización de masas. En ella la educación se concibe como una pieza fundamental de los procesos institucionalizados de producción social y cultural. Se caracteriza por la separación entre la teoría y la práctica, de forma que el educador se convierte en un ejecutor de las orientaciones de los planificadores, investigadores y teóricos. Su diseño es lineal, se apoya en la psicología conductista enfatizando lo medible y observable y su función educativa es la transmisión de información (Carr y Kemmis, 1988).

La perspectiva académica implica la formación de profesores en una rama del saber; por ende, la transmisión y presentación del contenido es fundamental. En este modelo de transmisión de conocimientos, lo relevante es preparar al educador en el dominio de la asignatura que imparte, para contar con expertos en la materia.
Muchos modelos universitarios le apuestan a esta perspectiva, aunque se encuentra fuertemente cuestionada desde el auge de las teorías cognitivas en la educación.

Son las últimas dos perspectivas las que son útiles para caracterizar los tipos y tendencias de los procesos que son de interés en este trabajo. La perspectiva de la investigación de la práctica para la transformación social considera al profesor como un profesional autónomo que reflexiona críticamente la práctica cotidiana, para comprender el proceso educativo en su contexto y lograr la emancipación a través del compromiso social. Aquí se incluyen las experiencias de acción socioeducativa de la pedagogía y todas las vertientes de la investigación acción.

En la perspectiva de la práctica reflexiva, el proceso educativo se concibe como una actividad compleja, singular, determinada por el contexto, cargada de contradicciones y conflictos productivos. La formación del docente se fundamenta en la investigación "para y a partir de la práctica" por lo que da prioridad a la reflexión sobre la experiencia del docente a través de instrumentos propios de la investigación. Esta perspectiva se genera en el proceso individual de conciencia y reflexión de la recuperación, del análisis y de la decisión de transformación de parte de un educador (Sañudo, 1997).

Estas dos perspectivas constituyen las tendencias de investigación para la transformación de la práctica que se consideran más potentes para la formación de docentes y su desarrollo o profesionalización. Ambas parten del con- 
vencimiento de la pertinencia de comprender el trabajo del profesional como educador(a)investigador(a). Enseguida se analizan ambas perspectivas de investigación de la práctica con más detalle.

\section{La perspectiva de la investigación acción}

A la perspectiva de la práctica reflexiva para la transformación social, de manera más corta, conocida y genérica, se le conoce como investigación acción. Esta tendencia no se origina dentro del ámbito educativo sino en el campo social y es posible encontrar evidencias de esta estrategia desde la década del setenta. La investigación acción se ha ido desarrollando y consolidando de tal manera que, conocer su evolución, sirve para comprender su sentido actual.

En el comienzo de la década del setenta, las aspiraciones de cambio social se vinculan a las posibilidades subjetivas y a los compromisos personales de los docentes; sin embargo, los académicos socialmente comprometidos asumen que no es posible poner en marcha procesos decisivos de transformación social por medio de la buena voluntad de cada persona. Se dan cuenta de que lo común es que los grupos en el poder presionen para que los sujetos se adapten a las decisiones tomadas unilateralmente y no ocasionen conflictos al modelo estructural establecido. Es primordial garantizar que los docentes no produzcan cambios que pongan en peligro el estado de las cosas (Geibler y Hege, 1997).
En contraste, esta propuesta de investigación social "consiste en permitir que las personas desempeñen un rol activo en la mejora de las condiciones de su existencia" (McKernan, 1999, p. 17); implica el control democrático de las condiciones sociales. Esta estrategia se observa con mucha fuerza en las comunidades rurales de Latinoamérica en las que se desarrollan condiciones comunitarias autogestivas con la mira puesta en la emancipación y en la conciencia del compromiso social. Como representantes de esta tendencia se pueden identificar a Oquist, Later y Schuter, entre otros. Muchos de ellos con tendencias marxistas y con importantes implicaciones políticas como parte de las estrategias y en los productos esperados.

En Europa, especialmente en Alemania, se desarrolla la teoría de la acción socioeducativa que evoluciona hasta convertirse más en una estrategia de intervención que de emancipación social, aunque en algunos casos se integran ambas con un solo propósito educativo. Este es un tema obligado en la discusión de la pedagogía social en Alemania y poco tratado en otros países en la década del ochenta del siglo pasado. Su "orientación normativa es la creciente emancipación o autonomía individual y social de la persona" (Geibler y Hege, 1997, p. 15). Este modelo determina su validez a partir de criterios vitales prácticos y no a partir de criterios científicos tradicionales; se trata de que el contenido del conocimiento derivado de estas estrategias sea confiable y válido en esas condiciones históricas.

Sus postulados se refieren a que los métodos deben ser adecuados a la persona y al objeto de estudio, ya que se orientan a los problemas 
y casos concretos de la praxis, de tal manera que se corresponden al problema constituido y a su condición histórica. Ante estas situaciones problemáticas, los actores, utilizando métodos e instrumentos de intervención pertinentes y flexibles, intervienen sistemáticamente en contextos determinados.

En el proceso, la reflexión de la práctica es una competencia clave para interpretar las experiencias producidas, de tal manera que "la reflexión de la propia praxis pedagógica-social es elemento constitutivo de las estructuras y procesos sociales" (Geibler y Hege, 1997, p. 226) y la reflexión significa la permanente confrontación con los presupuestos y condiciones de cada acción.

En este proceso metodológico, la intervención socioeducativa tiene como objetivo la emancipación o autonomía de los participantes, por esto no puede verse como independiente de la persona que interviene, puesto que la situación de intervención desencadena también afectación emocional del pedagogo social. Esta perspectiva, aunque tiene su origen en la visión de la investigación social, modifica su estrategia y se acerca a la acción educativa como tal, es decir, a los procesos formativos de los individuos. Es claramente identificable una manifestación actual de esta tendencia en algunos investigadores de la Universidad Autónoma de Barcelona, en España, como Sarramona, Melich o Jordán.

En Inglaterra, por su parte, las primeras publicaciones sobre este tema se pueden identificar al final de la década del setenta y del ochenta del siglo pasado. La investigación acción empieza a trasladarse a los espacios educativos en donde encuentra una nueva vitalidad entre la comunidad de profesores que están interesados en participar activamente en las decisiones y las acciones de mejora en su práctica o en las instituciones en las que se encuentran. Representantes de esto son teóricos como Stenhouse y Elliot, que se preocupaban por la situación educativa desde dentro.

Carr y Kemmis (1988) describen que esta tendencia se origina a partir de la oposición de académicos a la perspectiva técnica. Se presenta como humanística, democrática y racionalista, en el sentido de que todos tienen la oportunidad de participar en las decisiones prácticas de las acciones educativas. La investigación está articulada a las propuestas de mejora que los grupos de trabajo determinan de acuerdo con sus propios criterios. Las herramientas de investigación utilizadas recuperan, analizan y permiten la reflexión de los mismos grupos con respecto a su hacer. En cuanto a lo que produce, el conocimiento es propio de la comunidad y tiene un valor histórico y contextualizado.

Stenhouse, el más importante y conocido representante de esta tendencia, estableció la diferencia de investigar sobre la acción y en la acción. Investigar sobre la acción implica que el objeto, la práctica en este caso, es independiente del sujeto que la realiza, la indaga tomando distancia de ella lo más neutro y ajeno posible. Investigar en la acción implica la total involucración del investigador que está dentro de la práctica misma, es decir, es su acción misma la que indaga. Esta última idea fue la que dio 
origen a la investigación del docente sobre su práctica. Stenhouse propone al profesor como investigador para indicar la dependencia de la mejora educativa con su capacidad de indagary reflexionar su hacer en el aula. Sus principales intenciones son la comprensión y la emancipación profesional. Según Stenhouse (1996), la investigación perfecciona la docencia porque indaga sistemática y conscientemente de la práctica, desarrollando la capacidad crítica del docente y, a partir de ahí, su emancipación.

El aporte de Stenhouse, primero en Gran Bretaña y posteriormente en el mundo, detonó un movimiento de transformación de la práctica desde la investigación de la misma y no a partir de investigadores de gabinete ajenos al trabajo escolar cotidiano. Pero fue Elliot, colaborador de Stenhouse, quien formaliza las ideas en este sentido $y$, a partir de su trabajo, la investigación acción dirigida al currículo tiene un mayor alcance y repercusión; especialmente, es relevante su insistencia en que la investigación acción afecta primordialmente al proceso formativo del(a) estudiante.

La investigación en la acción, y no sobre la acción, es el tipo de proceso en el que el acto de indagar es necesariamente un acto sustantivo, entendido como un movimiento que posea sentido en un área específica de acción y se hace siempre tomando en consideración el aprendizaje de los estudiantes. "En educación los actos sustantivos -simplificando un tanto en exceso-se hallan concebidos para ayudar a las personas a aprender" (Elliot, 1996, p. 88). Esta condición es la que separa de manera definitiva el anterior proceso de investigación social y de investigación educativa.
En 1985, publica sus experiencias en cuanto a la investigación como base de la enseñanza, alimentando desde esta perspectiva el movimiento de reforma del currículo en las escuelas de esa década. La investigación la entiende como "una actividad sistemática y autocrítica" (Elliot, 1996, p. 28) en la que el profesor interpreta su práctica cotidiana a través de un proceso autorreflexivo en búsqueda de su desarrollo, ya que la tarea del profesional de la educación en ejercicio es mejorar la calidad de vida en una situación institucional educativa.

En su propuesta, desarrolla una comprensión interpretativa personal de los problemas de un grupo que investiga la práctica. Está constituida por una serie de hipótesis que son conclusiones provisionales, producto del proceso de la investigación, y han de comprobarse en la acción curricular en el aula. Este tipo de indagación se define como "el estudio de una situación social para tratar de mejorar la calidad de la acción misma" (Elliot, 1996, p. 88). Sus objetivos son proporcionar elementos que sirvan para facilitar el juicio práctico en situaciones concretas y mejorar la práctica. La producción y utilización del conocimiento se subordina a la mejora de la práctica, por lo que la validez de la teoría depende de su utilidad para ayudar a las personas a actuar de modo más inteligente.

El proceso de la investigación en la acción opera mediante el desarrollo reflexivo de la práctica educativa. El grupo de profesores modifican algún aspecto de la práctica docente como respuesta a algún problema práctico, revisando después su eficacia al resolverlo. Mediante la investigación se modifica la comprensión 
inicial del problema, por lo tanto, la decisión de adoptar una estrategia de cambio precede al desarrollo de la comprensión, producto de la investigación. La acción inicia la acción, en un proceso que representa la lógica natural del pensamiento práctico. No hay separación entre la investigación y la práctica; la práctica misma es la forma de investigación porque "la búsqueda de la comprensión se desarrolla a través de la modificación de la práctica y no antes de tales cambios" (Elliot, 1996, p. 38).

Los profesores pueden modificar su práctica, aprender sobre ella y producir conocimiento práctico, siempre de manera más pertinente que lo que produce la investigación de gabinete, ajena a la situación cotidiana del aula. Es ésta la razón de que el educador perciba estas tendencias como más cercanas a su práctica y le permita mejorar su hacer sin reproducir de manera prescriptiva las recetas propuestas acríticamente.

En concreto, Stenhouse y Elliot proponen lo que McKernan (1999) llama el modelo práctico de investigación acción. Su propósito es comprender la práctica y resolver problemas inmediatos. En ambas propuestas, la modificación de la práctica debe dirigirse a lo que se considera más educativo, aunque el modelo aspira a influir además en cómo las personas se comportan, piensan, sienten y viven, con una última intención moral.

En el desarrollo de la investigación acción en México, se destaca el trabajo de Fierro, Fortoul y Rosas que han sido fundamentales para el desarrollo de esta propuesta en este país. Su base es la investigación acción, especialmente aquella en la que pensaron los ingleses Stenhouse y Elliot. Entienden la práctica docente como una

\begin{abstract}
praxis social, objetiva e intencional en el que intervienen los significados, las percepciones y las acciones de los agentes implicados en el proceso -maestros, alumnos, autoridades educativas y padres de familia- así como los aspectos político institucionales, administrativos y normativos, que según el proyecto educativo de cada país, delimitan la función del maestro. (Fierro, Fortoul y Rosas, 1999, p. 21)
\end{abstract}

La propuesta se basa en el análisis crítico de la práctica conducente a reconocer las contradicciones presentes y a ubicarse en ella para entender, analizar y revisar el alcance de las acciones, siempre en función de los estudiantes. Este proceso implica un concepto de cambio que inicia modificando la percepción y el significado de la práctica y que genera posteriormente preguntas y proyectos que pueden ser llevados a cabo de manera sistemática.

La reflexión produce posibilidad de diálogo y en el ejercicio auténtico de este intercambio, el o la docente confronta sus ideas y sus convicciones con otros iguales, dando lugar al pensamiento crítico. Todo este proceso produce transformaciones en la práctica cotidiana. La estrategia se basa en el trabajo grupal y se desarrolla en el seno de un grupo de docentes que tienen la inquietud de superarse. Se les invita a realizar un análisis crítico de seis dimensiones (personal, interpersonal, social, institucional, didáctica y valoral) que conforman la complejidad de la práctica docente. 
Fierro, Fortoul y Rosas (1999) ponen especial énfasis en que el docente reflexione sobre su práctica y su contexto con indicadores y ejercicios precisos. Esta característica es especialmente interesante para los educadores que la encuentran accesible y utilizable. Aunque éste no es el único trabajo de este tipo en México, sí se puede identificar como el que más alcance ha tenido hasta la fecha.

De manera paralela a esta perspectiva, se pueden reconocer propuestas que, aunque en mucho comparten sus supuestos con la anterior, contienen elementos teóricos y operativos que las distinguen. Así, la llamada práctica reflexiva se desarrolla con elementos teóricos propios $\mathrm{y}$, aunque es más reciente, también tiene su historia.

\section{La perspectiva de la práctica reflexiva}

Aunque la reflexión y la investigación son operaciones constantes en lo descrito anteriormente, el énfasis está puesto en los efectos, es decir, en producir autonomía, emancipación y democracia a través de resolver en comunidad situaciones prácticas en el aula. En el caso de la perspectiva de la practica reflexiva, el foco es la operación misma de reflexionar sobre la práctica; claro está que sigue siendo importante el efecto, sin embargo, la transformación de la práctica es algo inherente en la recursividad del proceso.

Uno de los autores más conocidos y de mayor repercusión en esta línea es Schön, investiga- dor del Massachusetts Institute of Technology (MIT), que indaga y sistematiza una propuesta epistemológica sobre el conocimiento reflexivo en la práctica a partir de la reflexión desde la acción. Aunque este autor no se refiere en especial a los profesionales de la educación, su propuesta está referida al proceso formativo de profesionales universitarios. Revisa las competencias del profesor universitario para formar profesionales reflexivos.

Las afirmaciones que son lo suficientemente potentes para ser fundamentales en el ámbito de formación de educadores reflexivos, de hecho, han sido punto de partida para generar ensayos y estrategias para el análisis reflexivo de la práctica educativa. Su producción la realiza en la División para el Estudio y la Investigación para la Educación del MIT y hace referencia a trabajos que le sirven de fundamento como los de Hainer, Argyris y Rein, colegas del mismo instituto.

Propone una epistemología de la práctica basada en el análisis de las relaciones entre pensamiento y acción del profesional y entre ésta y los contextos personales e institucionales. Su trabajo tiene como génesis la crisis de confianza de la sociedad en las profesiones y también el declive de la auto imagen profesional, que parece tener sus raíces en un creciente escepticismo acerca de la efectividad profesional. De acuerdo con esto: "la práctica ingeniosa de un caso único parece anómala cuando la competencia profesional está modelada en términos de aplicación de las técnicas establecidas para sucesos recurrentes" (Schön, 1997, p. 30). 
El profesional técnico, producto de la formación universitaria y la profesional positivista, ha fracasado en dar cuenta de la competencia práctica en situaciones divergentes, por lo que Schön propone formar al profesional en la práctica reflexiva que desarrolla la habilidad de resolver situaciones de incertidumbre, inestabilidad, carácter único y conflicto de valores en el campo laboral. El proceso implica reflexionar sobre procedimientos que no podemos normalmente describir y de los que no se es muy consciente, es decir, desarrollar la habilidad de pensar en hacer, al mismo tiempo que podemos pensar mientras lo hacemos.

La investigación desde la práctica es similar a la investigación tradicional, sólo que el protagonista es otra vez el docente mismo. La mediación fundamental que permite el distanciamiento del docente con respecto a su práctica es la auto observación de su hacer en el aula, y el análisis realizado de las acciones presentes en el registro le permite generar conocimiento sobre lo que hace.

El método, en esta propuesta, es, entre otros y de manera prioritaria, la auto observación, ya que el investigador se observa a sí mismo, indaga sobre su acción para producir conocimiento práctico. En esta propuesta hablar del sujeto de investigación presupone la existencia del objeto por indagar, ya que es el sujeto el que lo nombra y lo modifica; al respecto dice Gutiérrez y Delgado (1993): "existe en la auto observación una superación de la paradoja separadora de sujeto y objeto." (p.157). Por su parte, Sañudo (2006) afirma:
Consecuentemente, el instrumento pertinente para la auto observación es el auto registro, que es una herramienta metodológica propia de una investigación cualitativa que tiene por objeto la transformación y mejora de la propia actividad del investigador. Para este trabajo, el auto registro, es un conjunto de elementos significativos recopilados, sintácticamente organizados que deben ser analizados, valorados y utilizados para construir presupuestos conceptuales que permitan mejorar la labor educativa.(p. 14)

El investigador de la práctica interactúa reflexivamente con la situación a través de los registros, produciendo nuevos significados que son a su vez enfrentados a nuevas confusiones e incertidumbres, reflexiona desde la acción y desarrolla una conciencia de su resistencia al cambio, permitiéndole darse cuenta cuándo las hipótesis que va formulando son o no adecuadas y por qué lo son. Por esto, debe actuar de acuerdo con lo que ha construido pero debe romperla más tarde para encontrar un nuevo sentido. Subyace en la reflexión en la acción una concepción constructivista que lleva a considerar al práctico como alguien que construye las situaciones de su práctica en todos los modos de su competencia profesional.

De acuerdo con lo anterior, la operación más relevante en este proceso es la reflexión que está al servicio del conocimiento y con objetivos prácticos; es decir, está en uso. Así, la reflexión elimina el espacio entre la investigación y su implementación: "aquí el intercambio entre la investigación y práctica es inmediato y la reflexión desde la acción es su propia implementación" (Schön, 1997, p. 270). Este proceso constituye lo que se denomina investigación reflexiva. 
Schön es una de las fuentes teóricas para importantes autores en la concepción y operación de la transformación ${ }^{2}$ de la práctica, como es el caso de Carr y Kemmis. No sólo utilizan para su entramado teórico a Schön sino que articulan una categoría social a través de la acción comunicativa de Habermas, que pertenece a la corriente de la teoría filosófica y social crítica de la Escuela de Frankfurt. Este planteamiento reitera la necesidad de la reflexión y la conciencia para lograr la emancipación de los sujetos, en este caso, de los educadores.

Al inicio de su trabajo, Carr, del Reino Unido, y Kemmis, australiano, se inscriben en la tendencia inglesa de la investigación acción, pero posteriormente hacen un esfuerzo por sistematizar lo que ellos denominan la teoría crítica de la educación, con la intención de poner las bases incipientes de lo que puede constituir una teoría de la educación como un área de conocimiento científico, caracterizado por ser un conocimiento práctico. La práctica es el constitutivo funcional de la educación y su indagación es la manera de conocerla y dinamizarla.

La orientación crítica, como la denominan Carr y Kemmis (1988), se caracteriza por ser democrática, dialéctica y emancipadora. Aunque social y políticamente determinada, la práctica se mejora y reformula mediante la reflexión y la deliberación crítica sobre ella; el educador es protagonista en la transformación del hecho

2 En mucha literatura referente a ese tema cualquiera de estas tendencias que investiga la práctica, sea social o reflexiva, se le llama investigación acción. Tal es el caso de MacKernan (1999). educativo. Los anteriores autores plantean un enfoque crítico-interpretativo-activista (McKernan, 1999) con un modo de investigación emancipador que produce conocimiento práctico sistematizado, el cual puede llegar a constituir una teorización educativa.

El proceso permite descubrir los significados interpretativos que tienen los profesores acerca de las acciones educativas y, posteriormente, los reorganiza para modificar la práctica. Se basa en las categorías críticas de los profesionales de la educación en ejercicio y, a partir de ahí, da prioridad a la crítica de la práctica a través de comunidades de comprensión auto reflexiva. Está basada en bucles recursivos de la acción humana que se patentizan en un plan progresivo de acción que incluye observación-acción-reflexión, siempre articulado a las relaciones institucionales y sociales en las que trabajan y viven. Así, la creación de teoría educativa es asunto de los profesionales de la educación en su conjunto.

\section{La investigación para producir conocimiento educativo}

Lo que realmente distingue a la investigación educativa de otros tipos y objetos de investigación es su utilidad y relevancia para los educadores. Estas dos condiciones no son tan simples como determinar el impacto y la influencia, sino que lo que exigen es una reconceptualización de lo que debe entenderse como lo educativamente importante, ya que la investigación educativa no es investigación sobre educación sino una investigación con un 
propósito educativo (Carr, 1996), la cual debe proveer de información útil y relevante para la educación y requiere ser comprendida en su conjunto como una acción que da cuenta del porqué se hace lo que se hace y de qué manera se hace.

Las ideas anteriores alimentan el trabajo de varios educadores españoles, que escriben de manera sistemática acerca de la reflexión de la práctica y la posibilidad de generar conocimiento educativo a partir de la indagación del hacer reflexivo del educador. Con base en la historia, aciertos y retrocesos, y en el marco que brindan Gimeno Sacristán, López Melero y Pérez Gómez (Universidad de Málaga), entre otros, se hace necesario encontrar una nueva racionalidad de la investigación educativa.

En este sentido, es fundamental para la educación un cuerpo teórico propio. No pensar ya en la educación como un espacio vacío en donde es necesario hacer converger diversos campos de conocimientos para poder explicarla, sino comprenderla como un conjunto de acciones articuladas específicas que conforman un campo de conocimiento concreto que requieren una teoría propia para ser explicitados, indagados e innovados (Sañudo, 2005).

La educación no es posible comprenderla desde una disciplina concreta ya construida. Lo educativo tiene una naturaleza propia y distintiva que comparte en algunos sectores con otras áreas del conocimiento que le apoyan para comprender ciertos eventos. Actualmente, es necesario reconocer que han empezado a "buscarse formas de superar las distancias entre la teoría de la educación y las prácticas que dan a toda esa teoría su razón de ser" (Carr, 1960, p. 53).

Anteriormente, esta naturaleza propia de la educación (la práctica) se entendía como una disciplina normativa en la que se aplicaban distintos principios teóricos y, por lo tanto, no podía tener el rango de teoría. Ahora se asume que no existe una teoría educativa independiente de la práctica educativa, sino que debe estar intrínsecamente relacionada con las experiencias de los educadores. La teoría no se aplica o se deriva de la práctica educativa sino que transforma la práctica, modificando las formas de comprenderla, por lo que, consecuentemente, mejoran la calidad de la participación de los educadores y les permite ejercer mejor (Sañudo, 2005).

De acuerdo con esto, el investigador no puede sustraerse de su compromiso para mejorar la educación y si es el educador el que investiga su práctica cotidiana, la mejora es inherente al proceso mismo de investigar.

La investigación es una estrategia de producción de conocimiento con diversos niveles de rigurosidad y alcance. El educador o un colectivo en una institución educativa tienen la opción de utilizar las estrategias de investigación para indagar y conocer su práctica, para intervenirla e innovarla o para aportar a la educación, a la política educativa o a la toma de decisiones desde la práctica misma. En esta modalidad, la distancia entre la investigación y su uso disminuye de manera definitiva. La investigación de la práctica garantiza la mejora educativa in situ. (Sañudo, 2006, p. 15) 
De acuerdo con López (2006), la investigación en educación requiere, además de las transformaciones en la práctica de los sujetos, el objeto de investigación o, en los contextos donde se investiga, la transformación de los propios protagonistas:

Es decir, que toda investigación, además de la evolución de los sujetos, categorías y contextos, requiere una reflexión sobre la metodología utilizada, una visión y revisión de los contenidos para señalar hacia donde nos dirigimos y qué nuevas perspectivas se nos abren desde nuestros hallazgos, así como los cambios y transformaciones de los propios investigadores: cambios ideológicos, epistemológicos, éticos, metodológicos, etc. Sólo así podremos decir que la investigación que realizamos es educativa. (López, 2006, p. 1)

En este sentido, la investigación de la práctica como actitud científica implica una orientación en la que este tipo de conocimiento o saber educativo es relativo a cualquier práctica, tiene una lógica de construcción y producción específica y tiene sus propias mediaciones en su indagación. La investigación educativa produce finalmente conocimiento propio de la educación, es decir, conocimiento práctico.

Entonces, el punto de partida no es la discusión de la posibilidad que tiene el docente de ser o no un potencial investigador, sino que está centrada en la cualidad de pertinencia de la investigación misma. Lo relevante para ser discutido tiene que ver con la índole educativa de la investigación sobre y en la educación y la afirmación de que el compromiso ético de los in- vestigadores es incidir, a través de los procesos y resultados de su trabajo, en la mejora de la educación en general, pero especialmente de la práctica educativa (Sikes, Nixon y Carr, 2003).

La investigación no sólo provee de evidencia a los prácticos y a los diseñadores de políticas, sino además es una fuente pública de interpretaciones de las evidencias de temas específicos, que debe comprometer al público en un debate reflexivo acerca de los fines y propósitos de la educación. Tiene el compromiso social de producir una práctica reflexiva que busca necesariamente mejorar la educación y la sociedad (Sañudo, 1997).

\section{Referencias}

Barbier, J. (1993). La evaluación en los procesos de formación. Barcelona, España: Paidós.

Cabero, J. (2001). Tecnología educativa. Diseño y utilización de los medios de enseñanza. Barcelona, España: Paidós.

Carr, P. (1960). Night of stars. New York, USA: Intermedial Editions.

Carr, W. y Kemmis, S. (1988). Teoría crítica de la enseñanza. La investigación acción en la formación del profesorado. Barcelona, España: Martínez Roca. 
Carr, W. (1996). Una teoría para la educación. Hacia una investigación educativa crítica. Madrid, España: Morata.

Elliot, J. (1996). El cambio educativo desde la investigación acción. Madrid, España: Morata.

Fierro, C., Fortoul, B. y Rosas, L. (1999). Transformando la práctica docente. Una propuesta basada en la investigación acción. México: Paidós.

Geibler, K. A. y Hege, M. (1997). Acción socioeducativa. Modelos, métodos y técnicas. Madrid, España: Narcea.

Gimeno, J. (1994). El currículo: una reflexión sobre la práctica (4a. ed.). Madrid, España: Morata.

Gimeno, J. (1998). Poderes inestables de la educación. Madrid, España: Morata.

Gimeno, J. y Pérez, A. (1997). Comprender y transformar la enseñanza (6a. ed.). Madrid, España: Morata.

Gutiérrez, J. y Delgado, J. M. (1995). Teoría de la observación. En J. M. Delgado y J. Gutiérrez (Eds.), Métodos y técnicas cualitativas de investigación en ciencias sociales. Madrid, España: Síntesis.
Habermas, J. (1989). La acción comunicativa (Vol. 1). Buenos Aires, Argentina: Taurus.

Hargreaves, A. (1996). Profesorado, cultura y posmodernidad (Cambian los tiempos, cambian los profesores). Madrid, España: Morata.

Lonergan, B. (1989). Dimensiones del significado. (En L. Sánchez Villaseñor (Ed. y Trad.), Textos escogidos. Guadalajara, México: ITESO.

López, M. (2006). La investigación en educación como fundamento de nuestra práctica. Documento interno de la Universidad de Málaga.

McKernan, J. (1999). Investigación-acción y currículum. Madrid, España: Morata.

Sacristán, J. (1997, agosto). El Conocimiento Necesario: Teórico o Práctico. Novedades Educativas, 9 (80).

Salgueiro, A. (1998). Saber docente y práctica cotidiana. Barcelona, España: Octaedro.

Sañudo, L. (1997) "Una experiencia sobre la transformación de la práctica docente", en En torno a la intervención de la práctica educativa de Campechano et al. Guadalajara, UNED. 
Sañudo, L. (2005, enero-junio) Hacia la Definición de la Teoría Educativa: Posibilidades Desde la Hermenéutica. Revista Red de Posgrados en Educación, (2), 8-22.

Sañudo, L. (2006). El proceso de significación de la práctica como sistema complejo. En M. Perales (Ed.), La significación de la práctica (pp. 17-54). México: Paidós.

Schön, D. (1992). La formación de profesionales reflexivos. Hacia un nuevo diseño de la enseñanza y el aprendizaje en las profesiones. Barcelona, España: Paidós.
Schön, D. (1990). El profesional reflexivo. Cómo piensan los profesores cuando actúan. Barcelona, España: Paidós.

Sikes, P., Nixon, J. y Carr, W. (Eds.). (2003). The moral foundations of educational research. Knowledge, inquiry and values. Maidenhead, Berkshire, England: Open University Press.

Stenhouse, L. (1996). La investigación como base de la enseñanza. Madrid, España: Morata. 